\title{
A MICROMACHINED INKING CHIP FOR SCANNING PROBE NANOLITHOGRAPHY USING LOCAL THERMAL VAPOR INKING METHOD
}

\author{
Shifeng Li, Kashan A Shaikh, Sandra Szegedi, Edgar Goluch and Chang Liu \\ Micro and Nanotechnology Laboratory, University of Illinois at Urbana and Champaign \\ 208 North Wright Street, Urbana, IL, USA
}

\begin{abstract}
A new method for inking scanning probes, based on local thermal evaporative inking transfer, has been demonstrated in this paper. The new method results in low loss and rapid parallel inking action. The scanning probes are accommodated at the opening of each ink reservoir. A underneath resistive heater is provided local heating to initiate vaporization of inks to uniformly coat individual probe. $1 \mathrm{mM}$ 16-Mercaptohexadecanoic acid (MHA) in the ethanol solution is used to characterize this inking chip. After 2 minutes heating and following 2 minutes cooling, MHA patterns are successfully written on the fresh gold-coated silicon substrate at $25{ }^{\circ} \mathrm{C}$ room temperature and $30 \%$ relative humidity environment. The minimum feature is less than $60 \mathrm{~nm}$. Moreover, the different ink channels can be loaded different inks so this chip has multiple inking capabilities potential. Also, due to special design of ink reservoirs, each probe is completely sealed inside individual reservoir so the ink cross contamination is limited Keywords: Scanning Probe Nanolithography, Micromachining, Surface Tension and Reservoir Array
\end{abstract}

\section{INTRODUCTION}

Scanning Probe Nanolithography (SPL) has been widely used to generate nanometer scale pattern on the various substrates [1-3]. The chemicals (inks) need deposit on scanning probe tips for subsequent writing onto a substrate $[\mathbf{4 , 5}$. The inking is a very critical and challenging step. Ideally, the inking procedure should be fast, highly efficient, and paralleled ink transfer onto an array of SPL probes. Existing methods of inking face severe limitations. The liquid phase dip inking is simple and popular, but it provides non-uniform, uncontrollable inking. It also suffers from high rate of evaporative ink loss from reservoirs and cross-contamination [6]. Inkpad based probe inking method is proposed to overcome this issue. It employs a porous membrane (e.g., PDMS) for containing/capping the inking solution. However, it takes long time ( $>6$ hours) for thiol molecules to diffuse through the thin membrane from ink-delivery channels [7]. Vapor-phase inking is uniform, reliable, and much faster than liquid phase inking. Traditionally, vapor phase inking is conducted by placing probes in a container filled with liquid chemical solutions or crystallized chemical compounds. Unfortunately, this method does not support multi-probe and multi-ink delivery [8].

We report a new method for inking scanning probes, including the design, fabrication, and testing of the new chip and the validation of performance. The new method, based on local thermal evaporative inking transfer, results in low loss and rapid parallel inking action (minutes). The inks are delivered to the closely spaced ink sites that provide local, on-demand vaporization and ink transfer to the scanning probes.

\section{DESIGN AND FABRICATION}

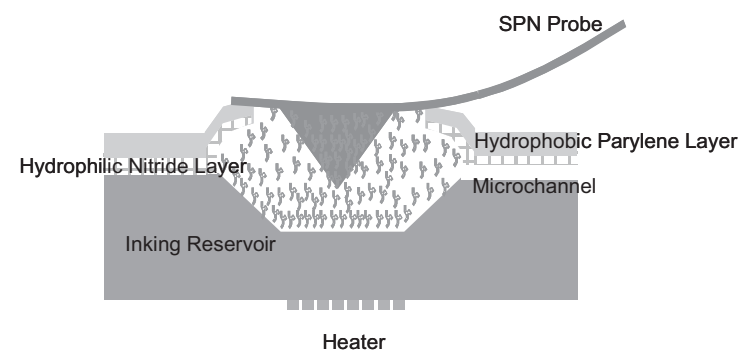

(a)

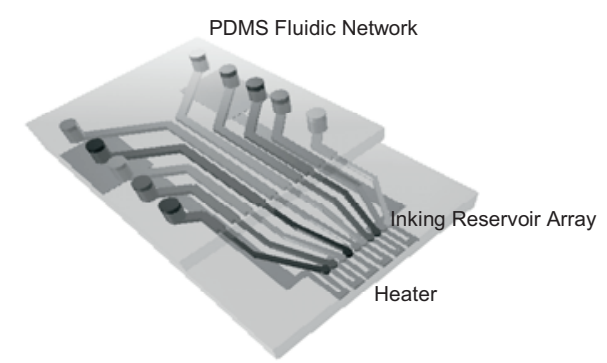

(b)

Figure 1. (a) Mechanism of local thermal vapor inking for SPL (b) Schematic of local thermal vapor inking based inking chip for SPL

This novel inking chip consists of two parts: PDMS fluidic network and silicon inking reservoir array. The ink loading channels are made inside PDMS layer using softlithogrpahy. The inking channels are made of hydrophilic silicon nitride using the standard micromachining techniques. Also, in order to keep ink solution from overflowing the reservoirs, a $2-\mu \mathrm{m}$ thick highly hydrophobic Parylene layer is deposited on the top of silicon nitride layer. Each ink reservoir is designed to accommodate each SPL probe. A thin film heater is fabricated underneath the silicon chip to locally heat up thiol molecules inside reservoirs (Fig. 1a). At first, the specific ink is delivered into the specific inking channel through PDMS fluidic network. Due to the dominant surface tension force inside hydrophilic silicon nitride microchannels, the thiol solution would be automatically pumped into individual ink reservoirs. After the probes are located on the right inking reservoirs, the temperature is locally increased by applying the current to the underneath thin film heater. When the temperature is up to the certain melting point $\left(>60{ }^{\circ} \mathrm{C}\right)$, the deposited thiol molecules inside each ink reservoir are evaporated and uniformly coated on the tip of SPL probe (Fig. 1b). 


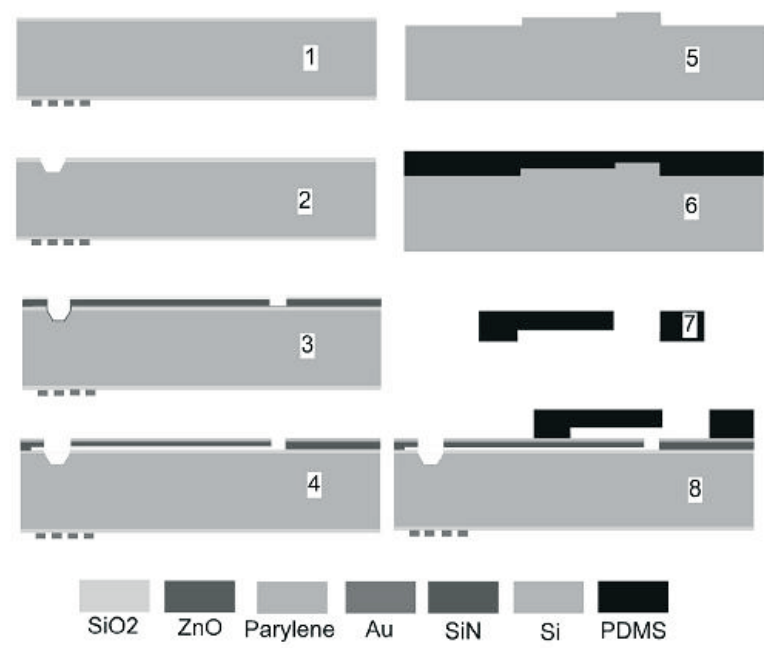

Figure 2. Inking chip fabrication flow: (1) Au thin film heater fabrication on oxide wafer; (2) flat bottom cavity array EDP etching; (3) scarification layer $\mathrm{ZnO}$ deposition and patterning and function layers PECVD nitride / Parylene deposition, then RIE etching windows;(4) hydrochloride acid solution chip releasing; (5) Silicon molding surface treatment;(6) PDMS liquid polymer heat curing; (7) loading hole punching and cutting; (8) PDMS layer alignment with silicon chip

Many advantages are associated with this novel inking chip: (1) no active pumping or vavling is required to transport ink solution and inks transport in the microchannel is mainly due to automatically surface tension force, (2) it is low loss and rapid parallel uniform inking, (3) the cross contamination of adjacent ink reservoir is limited because of the special inking reservoir design, (4) it is scalable to high density parallel inking, (5) it has multiple ink coating capability due to the fact that the different microchannels can be filled different inks.

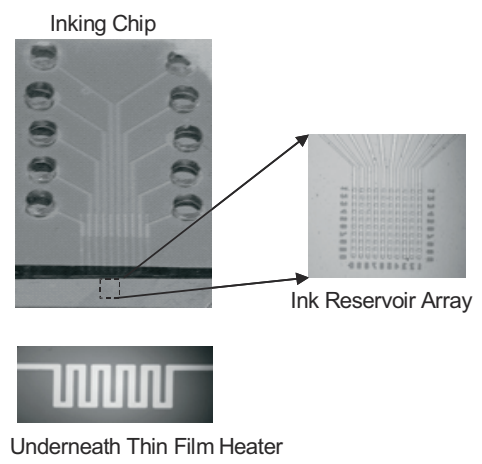

Figure 3. The assembled thermal vapor-inking chip

The fabrication process to realize the novel-inking chip is shown in Fig. 2. (1) First, a 2600-A thick silicon dioxide layer is thermally grown on the 2" $<100>$-orientation silicon wafer (International Wafer Service, Portola Valley, CA, USA). A Cr/Au thin film resistive heater is deposited on the substrate using thermal evaporation $(\mathrm{Cr} / \mathrm{Au}=50 \mathrm{~A} / 5000 \mathrm{~A})$. (2) Using the photolithography with backside alignment, the $\mathrm{SiO}_{2}$ layer at the front side is wet etched to open square windows to etch $5 \mu \mathrm{m}$ depth reservoir cavities using EDP silicon etchant. (3) A $1 \mu \mathrm{m}$ thick $\mathrm{ZnO}$ is sputtered on the substrate as sacrificial layer, then the ink channels are photolithographically patterned. A $2-\mu \mathrm{m}$ thick silicon nitride layer and a $2-\mu \mathrm{m}$ Parylene layer are deposited to cover the ink microchannels, respectively. A 1200-A thick Al thin film is deposited as etching mask for subsequent reactive ion etching to etch inking loading holes and ink reservoir windows. (4) The whole chip is released using diluted $\mathrm{HCL}$ solution $(38 \% \mathrm{HCl}$ : $\mathrm{H}_{2} \mathrm{O}=2 \mathrm{ml}: 400 \mathrm{ml}$ ) for 48 hours shaking on an orbit shaker. The rotation speed of the shaker is set as 60 RPM. (5) The silicon mold is etched using deep reactive ion etching. The depth of mold pattern is $60 \mu \mathrm{m}$. (6) Before the molding, the silicon mold was deposited a thin carbon layer to enhance PDMS layer releasing, then PDMS prepolymer (10:1 mixing ratio with curing agent, Dow Corning Sylgard 184, Midland, MI, USA) is poured on the silicon mold. (7) After 30 minutes curing at $90{ }^{\circ} \mathrm{C}$, PDMS layer is peel off and punch the access holes using a sharpened needle. The blade cuts the PDMS layer into final dimensions: $30 \mathrm{~mm}$ wide and $40 \mathrm{~mm}$ long. (8) Finally, the PDMS layer is aligned and assembled with silicon chip. Fig. 3 is an optical picture of the final assembled inking chip.

\section{TEST RESULTS}

Thiol chemical MHA is used to characterize this inking chip. At first, $1 \mathrm{mM}$ ethanolic MHA is filled into PDMS channels. Once the ink solution reaches the loading holes at the beginning of the silicon nitride microchannels. Due to dominant surface tension force inside hydrophilic silicon nitride microchannels, the ink solution is automatically pumped to individual ink reservoir. Finally, due to quickly evaporation nature of ethanol, MHA ink is deposited inside ink reservoirs (Fig. 4). The measured resistance of the thin film heater is around $150 \Omega$. After applied $20 \mathrm{DC}$ voltages using a power supply (E3612A, Agilent, CA, USA), we found the local temperature at the ink reservoirs quickly reached MHA melting point $\left(64{ }^{\circ} \mathrm{C}\right)$ within 2 minutes. The inking chip subsequently cooled down for another 2 minutes to make sure uniform coating on the scanning probe tips (Fig. 5). After 2 minutes heating and following 2 minutes cooling, the inked probe (type A, NanoInk Inc. Chicago, USA) is loaded on Nscriptor (Nanoink Inc, Chicago, USA). MHA patterns are successfully written on the fresh gold-coated silicon substrate $(\mathrm{Au} / \mathrm{Cr}=30 \mathrm{~nm}$ / $5 \mathrm{~nm}$ ) at $25{ }^{\circ} \mathrm{C}$ room temperature and $30 \%$ relative humidity environment (Fig. 6a and 6b). The minimum feature is less than 60 $\mathrm{nm}$. In order to prove that the ink transfer is mainly due to the thermal evaporation, the tip of the scanning probe was sealed inside the ink reservoir and the thin film heater did not turn on After 15 mins inking, the probe was loaded on Nscriptor to write the pattern on the fresh gold-coated substrate under the same working temperature and humidity. It was found the same patterns written on the substrate at the least 10 times slower even after 15 minutes inking.

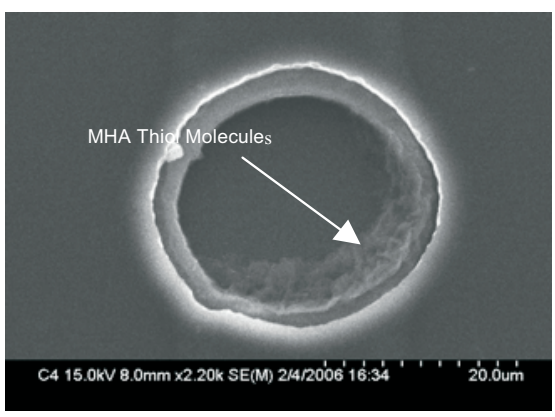

Figure 4. The chemical thiol was deposited inside individual ink reservoir 
The ink reservoir is specially designed as Fig. 1(a) and the tip of each SPL probe was sealed inside individual reservoir, so the ink cross contamination is expected to be limited. A crosscontamination test was performed by filled the ink solution in the specific ink channel as shown in Fig. 3 and leave the adjoined ink channels were empty. After using the aforementioned method to finish inking, we perform writing on fresh gold substrates. But only the probe, which is inked from the filled channel, can write the patterns on the substrate. For other probes, no MHA patterns were detected. We can conclude that no appreciable cross contamination occurs during the local thermal vapor inking.

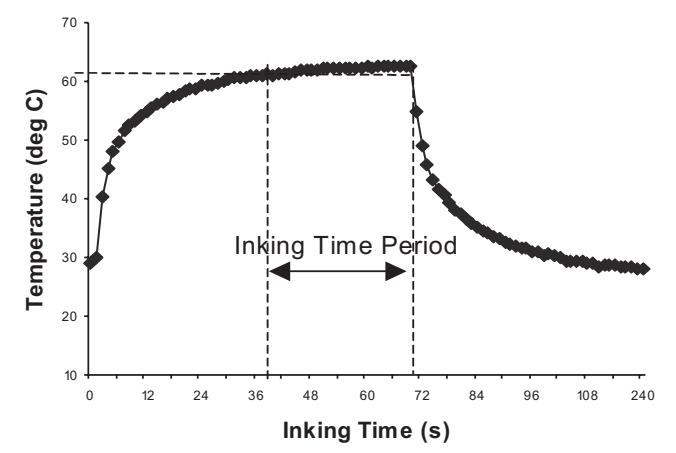

Figure 5. Transient temperature measurement during local thermal vapor inking using IR microscope
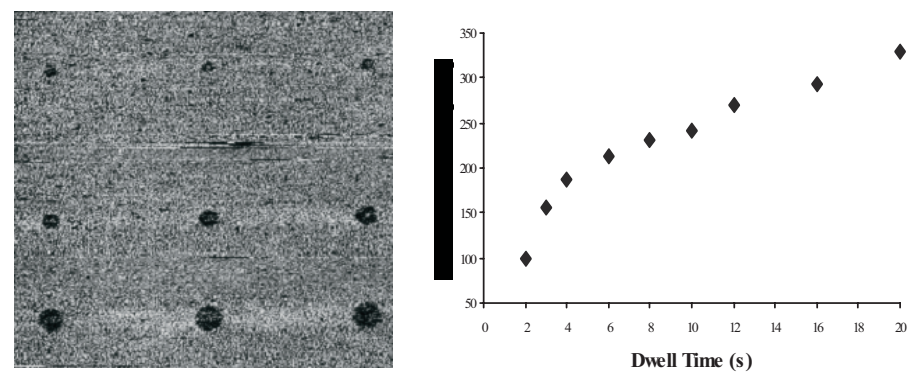

(a)
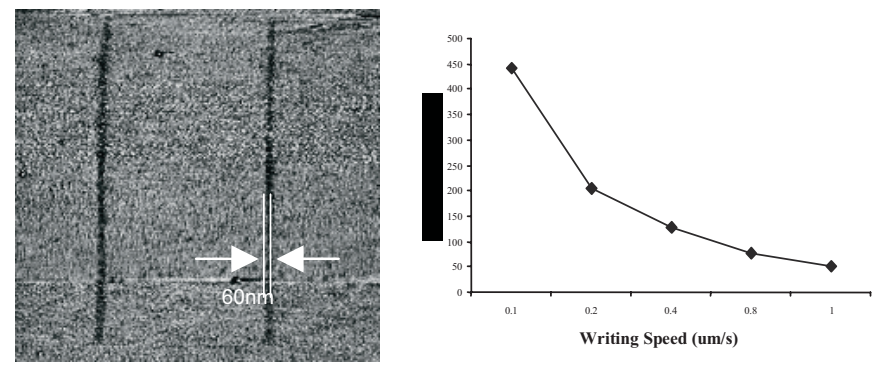

(b)

Figure 6. Characterization of local thermal vapor inking chip (a) dots array writing (3.58 $\mu \mathrm{m}$ by $3.58 \mu \mathrm{m}$ scanning size) and (b) lines writing (3.3 $\mu \mathrm{m}$ by $3.3 \mu \mathrm{m}$ scanning size) at $25{ }^{\circ} \mathrm{C}$ and relative humidity $30 \%$

\section{CONLUSION}

In this paper, we report a novel method to ink scanning probes for nanolithgraphy based on local thermal vapor ink transfer method. This method can finish low loss and parallel inking scanning probes within several minutes. At the same time, the special design ink reservoir can accommodate probe tip into individual reservoir to limit the possible ink contamination. Moreover, the different ink channels can be loaded different inks so this chip has multiple inking capabilities.

\section{ACKNOWLEDGEMENT}

The authors thank DARPA Advanced Lithography program and NSF Center for Nanoscale Science and Engineering (Northwestern University) for support.

\section{REFERENCES}

[1] L. Demers, D. Ginger, S. Park, S. Chung and C. Mirkin, "Direct Patterning of Modified Oligonucleotides on Metals and Insulators by Dip-Pen Nanolithography", Science, Vol. 296, pp. 1836-1838 (2002)

[2] K. Lee, J. Lim and C. Mirkin, "Protein, Nanosturctures Formed Via Direct-Write Dip-Pen Nanolithography" Am. Chem. Soc, Vol.125, pp.5588-5589, 2003

[3] H. Zhang, Z. Li and C. Mirkin, "Dip-Pen NanolithographyBased Methodology for Preparing Arrays of Nanosturctures Functionalized with Oligonucleotides" Adv. Mater. Vol. 16, pp.1480-1484, 2002

[4] J. Jang, S. Hong, G. Schatz and M. Ratner, "Self-assembly of Ink Molecules in Dip-Pen Nanolithography: A Diffusion Model", Journal of Chemical Physics, Vol. 115, pp. 2721-2729, 2001

[5] B.L. Weeks, A. Noy, A. E. Miller and J. J. DeYoeo, Physical

- Review Letters, Vol. 88, pp. 255505-1 to 255505-4 (2002)

[6] D. Banerjee, "Next Generation Microfluidic Ink Delivery Systems for Dip-Pen Nanolithography Application in Biotechnology" ASME Biomedicine Miniaturization, Irvine, CA, 2005

[7] K. Ryu, X. Wang, K. Shaikh, D. Bullen and E. Goluch, "Integrated Microfluidic Inking Chip for Scanning Probe Nanolithography" Applied Physics Letter, Vol. 85 pp. 136-138, 2004

[8] X. Wang, PhD Dissertation, "Microelectromechanical and Microfluidic Systems for Scanning Probe Lithography", University of Illinois at Urbana and Champaign, 2005 